\title{
KSHV seroprevalence, and blood and saliva viral loads in the HIV-infected population of south Texas
}

\author{
M Guadalupe *1,2, Y Flahive ${ }^{1}$, S Westbrook ${ }^{3}$, S Redding3,5, D Bullock 6 , \\ V Sankar³, B Agan7, S Barbieri3 ${ }^{3}$, CK Yeh ${ }^{3}$, H Dang ${ }^{4}$ and SJ Gao ${ }^{1,2}$
}

\begin{abstract}
Address: ${ }^{1}$ Greehey Children's Cancer Research Institute, University of Texas Health Science Center at San Antonio, San Antonio, Texas, USA, ${ }^{2}$ Department of Pediatrics, University of Texas Health Science Center at San Antonio, San Antonio, Texas, USA, ${ }^{3}$ Dental and Diagnostic Science, University of Texas Health Science Center at San Antonio, San Antonio, Texas, USA, ${ }^{4}$ Community Dentistry, University of Texas Health Science Center at San Antonio, San Antonio, Texas, USA, ${ }^{5}$ Dental Hygiene, University of Texas Health Science Center at San Antonio, San Antonio, Texas, USA, ${ }^{6}$ Medicine, University of Texas Health Science Center at San Antonio, San Antonio, Texas, USA and ${ }^{7}$ San Antonio Military Medical Center, San Antonio, Texas, USA

* Corresponding author
\end{abstract}

from II th International Conference on Malignancies in AIDS and Other Acquired Immunodeficiencies (ICMAOI): Basic, Epidemiologic, and Clinical Research

Bethesda, MD, USA. 6-7 October 2008

Published: 17 June 2009

Infectious Agents and Cancer 2009, 4(Suppl 2):P20 doi:10.1 I86/1750-9378-4-S2-P20

This abstract is available from: http://www.infectagentscancer.com/content/4/S2/P20

(C) 2009 Guadalupe et al; licensee BioMed Central Ltd.

Kaposi sarcoma (KS) is the most common neoplasm in HIV-infected subjects, and is associated with infection by Kaposi's sarcoma-associated herpesvirus (KSHV). Our previous studies have shown that KSHV epidemiology in South Texas is distinct with an increased KSHV seroprevalence in blood donors and unique distribution of KSHV genotypes in KS subjects. However, KSHV epidemiology in the HIV-infected population in South Texas remains undefined.

In this cross sectional study, we examined specific antibodies to KSHV latent nuclear antigen (LANA) by immunofluorescence antibody assay (IFA) and to KSHV lytic antigen ORF65 by ELISA in 25 HIV-infected KS subjects, 314 HIV-infected subjects, and 335 HIV-negative subjects. Relative antibody titers to ORF65 were estimated based on optical density values (O.D.). Blood and saliva viral loads were also determined in KSHV-seropositive subjects by quantitative real-time PCR. Antibodies to KSHV antigens were detected in 25 (100\%) KS subjects, 114 (36\%) HIV-infected subjects and 65 (19\%) HIV-negative subjects. KS subjects had higher antibody titers to ORF65 compared to HIV-infected and -negative subjects (median O.D. 0.76 versus 0.47 and $0.27 ; p=0.0017$ and 0.0001 , respectively). Antibody titers in HIV-infected subjects were also significantly higher than HIV-negative subjects $(p=0.0001)$.

Among the HIV-infected subjects, males had higher KSHV seroprevalence than females ( $42 \%, 95 \%$ CI: $35.6-47.4 \%$ versus $8 \%$, 95\% CI: $0.5-15.8 \%, p<0.0001)$. Compared to subjects with $>400$ CD4+ T cell counts, those with $<200$ $\mathrm{CD} 4+\mathrm{T}$ cell counts had higher KSHV seroprevalence (OR $=2.59,95 \%$ CI: $1.4-4.80, p=0.017)$ and higher antibody titers (median O.D. 0.48 versus $0.32 ; p=0.001$ ). KSHV blood and saliva viral loads were detected in $12(48 \%)$ and $9(36 \%)$ of $25 \mathrm{KS}$ subjects, $16(26 \%)$ and $12(19 \%)$ of 62 HIV-positive KSHV-seropositive subjects, and none of the HIV-negative KSHV-seropositive subjects, respectively. Among the KS subjects, KSHV blood and saliva viral loads were detected more frequently in those with active KS than those without active KS $(81.8 \%, 95 \%$ CI: $48.2-$ $97.7 \%$ and $54.5 \%, 95 \%$ CI: $23.3-83.2 \%$ versus $18 \%, 95 \%$ CI: $2.2-51.7 \%$ and $45 \%, 95 \%$ CI: $16.7-76.6 \%, \mathrm{p}=0.004$ and 0.05 , respectively). Together, these results indicate that HIV infection and the status of HIV disease might modulate KSHV infection and replication, and impact the development of KS. 